\title{
Application Analysis of Fuzzy Mathematics Based on Comprehensive Evaluation in Mathematical Modeling
}

\author{
Wu Wang \\ Zhonghuan Information College Tianjin University of Technology, Tianjin 300380, China
}

Keywords: Fuzzy mathematics, Mathematical modeling, Uncertainty

\begin{abstract}
Fuzzy mathematics is a science to study and deal with fuzzy phenomena, which reveals an uncertainty in division caused by the intermediate transition of differences between objective things. The significance of mathematical modeling lies in the application of mathematical theory to practical problems. As a relatively new theory, fuzzy mathematics has its own huge application background. The significance of mathematical modeling lies in the application of mathematical theory to practical problems. As a new theory, fuzzy mathematics has its huge application background. By introducing the fuzzy mathematical method into the mathematical modeling, we can get some mathematical models which are simpler in calculation and more suitable for the actual situation. Based on the comprehensive evaluation method, this paper discusses the application of fuzzy mathematics in mathematical modeling.
\end{abstract}

\section{Introduction}

Fuzzy mathematics, as a new subject to study how to deal with various fuzzy problems in different fields, is quite necessary for many fields of science and technology as well as managers [1] Fuzzy mathematics is a science to study and deal with fuzzy phenomena. What it reveals is an uncertainty in division caused by the intermediate transition of differences between objective things [2]. Fuzziness originates from the concept of fuzziness, which refers to a concept with certain connotation but no definite extension. There are many methods of fuzzy mathematics, such as fuzzy programming, fuzzy optimization design, comprehensive evaluation, fuzzy clustering analysis, fuzzy sorting, etc. The significance of mathematical modeling lies in the application of mathematical theory to practical problems. As a relatively new theory, fuzzy mathematics has its own huge application background [3]. People cannot change the objective laws determined by the characteristics of these disciplines in order to accommodate the existing teaching methods, but can only transform mathematics to make it more widely used. Fuzzy mathematics is formed in this context. [4]

The significance of mathematical modeling lies in the application of mathematical theory to practical problems, and fuzzy mathematics, as a newer theory, has its own huge application background. The introduction of fuzzy mathematical methods in mathematical modeling can obtain some mathematical models that are simpler to calculate and more in line with the actual situation [5]. The mathematical modeling competition for college students is a major extracurricular science and technology activity in colleges and universities. It is conducive to cultivating students 'ability to analyze problems and solve practical problems, as well as students' spirit of innovation and cooperation, which is conducive to expanding the application of mathematics in real life. The purpose of the modeling competition is to stimulate students 'enthusiasm for learning mathematics, improve students' comprehensive ability to build mathematical models and use computer technology to solve practical problems, develop knowledge, and cultivate creativity and cooperation consciousness [6]. This article mainly discusses the application of fuzzy mathematics in mathematical modeling based on comprehensive evaluation methods. 


\section{Comprehensive Evaluation Model Using Fuzzy Mathematics Method in Mathematical Modeling}

Due to the particularity of fuzzy mathematics theory, it is different from traditional mathematics theory. It is difficult for students to understand the essence of fuzzy mathematics and to deeply understand and consolidate the basic theories, principles and methods of fuzzy mathematics. The traditional evaluation system is still based on written examination, which cannot really examine the students' learning situation and lacks practical teaching evaluation and assessment links. Multiobjective programming is a kind of optimization problem. Because it has multiple objectives and requires each objective to obtain a better value at the same time, the solution method and process are relatively complex.

The so-called comprehensive evaluation is to make a general evaluation of things or objects restricted by various factors. There are two simplest methods for comprehensive evaluation. Let the evaluation object have $\mathrm{m}$ factors, give a score Si for each factor, and calculate the sum of the scores obtained by the evaluation object:

$$
\sum_{i=1}^{m} a_{i}=1
$$

So there are:

$$
S=\sum_{i=1}^{m} a_{i} S_{i}
$$

Let $U=\left(u_{1}, u_{2}, \ldots, u_{n}\right)$ be a set of $\mathrm{n}$ kinds of factors that measure the operation effect, called factor set. $V=\left(v_{1}, v_{2}, \ldots, v_{m}\right)$ is a set of $\mathrm{M}$ evaluations, called evaluation set. In general, the influence degree of each factor on the evaluation of operation effect is inconsistent, so the weight distribution of each factor is recorded as follows:

$$
A=\left(a_{1}, a_{2}, \ldots, a_{n}\right)
$$

Where $\sum_{i=1}^{n} a_{i}=1$, ai represents the weight of the i-th factor. In addition, $M$ evaluations are not all absolute positive and negative, so comprehensive evaluations should also be recorded as follows:

$$
B=\left(b_{1}, b_{2}, \ldots, b_{n}\right)
$$

Among them, $b_{j}(j=1,2, \ldots, m)$ reflects the position of the $\mathrm{j}$-th evaluation in the total evaluation set $\mathrm{V}$.

The factor set $U=\left(u_{1}, u_{2}, \ldots, u_{n}\right)$ is divided into s subsets according to some attributes:

$$
U_{i}=\left\{u_{i 1}, u_{i 2}, \ldots, u_{i n_{i}}\right\}
$$

A comprehensive evaluation is made for each sub factor set. The evaluation set is set as $V=\left(u_{1}, u_{2}, \ldots, u_{m}\right)$, and the weight distribution of each factor in Ui is as follows:

$$
A_{i}=\left\{a_{i 1}, a_{i 2}, \ldots, a_{i n_{i}}\right\}
$$

Of them, $\sum_{t=1}^{n_{i}} a_{i_{t}}=1$. If Ri is a single factor matrix, the first level evaluation vector is obtained 


$$
B_{i}=A_{i} \circ R_{i}=\left(b_{i_{1}}, b_{i_{2}}, \ldots, b_{i_{m}}\right)
$$

Each Ui, as a part of $\mathrm{U}$, reflects some of its attributes, and can assign weights $A=\left(a_{1}, a_{2}, \ldots, a_{s}\right)$ according to their importance, and then get the secondary evaluation vector:

$$
B=A \circ R=\left(b_{1}, b_{2}, \ldots, b_{m}\right)
$$

If each sub-factor set $U_{i}(i=1,2, \ldots, s)$ still contains more factors, Ui can be divided again.

\section{Multi-Objective Linear Programming Problem}

Mathematical knowledge is the result of cognition. Mathematical thought is the basic viewpoint in cognition activities. Mathematical methods provide ideas, logical means and operating principles for mathematical activities. There are qualitative differences between them. In this infinitely complex world, any set or sets of axioms cannot exhaust all relationships between things. This is not a philosophical conjecture, but a mathematical theorem.

Multi-objective linear programming has two or more objective functions, and the objective functions and constraints are all linear functions. Its mathematical model is expressed as follows:

$$
\max \left\{\begin{array}{l}
z_{1}=c_{11} x_{1}+c_{12} x_{2}+\ldots+c_{1 n} x_{n} \\
z_{2}=c_{21} x_{1}+c_{22} x_{2}+\ldots+c_{2 n} x_{n} \\
\ldots \\
z_{n}=c_{n 1} x_{1}+c_{n 2} x_{2}+\ldots+c_{n n} x_{n}
\end{array}\right.
$$

The constraints are:

$$
\left\{\begin{array}{l}
a_{11} x_{1}+a_{12} x_{2}+\ldots+a_{1 n} x_{n} \leq b_{1} \\
a_{21} x_{1}+a_{22} x_{2}+\ldots+a_{2 n} x_{n} \leq b_{2} \\
\ldots \\
x_{1}, x_{2}, \ldots, x_{n} \geq 0
\end{array}\right.
$$

In the specific solution, a compromise solution needs to be adopted to make each objective function as large as possible. The fuzzy mathematical programming method can fuzzify each of its objective functions, transform a multi-objective problem into a single objective, and thus obtain a fuzzy optimal solution to the problem.

\section{The Significance of Fuzzy Mathematics to the Construction of Mathematical Modeling Teaching System}

Although mathematical thinking methods are specifically reflected in basic knowledge, the teaching of mathematical knowledge cannot replace the teaching of mathematical thinking methods. Teachers should combine the actual situation of students, constantly update the teaching content, redesign the teaching process, pay attention to the cause and effect of problem analysis, and reveal the actual source and background of various fuzzy concepts. By fuzzifying the objective function, the multi-objective problem can be transformed into a single objective, which can be easily solved by means of tool software [7]. Mathematics teaching provides an important means for students to grasp the whole of mathematics knowledge, and enables students to form a unified concept. Students can present an overall image in their minds when thinking about mathematical problems, especially complicated mathematical problems.

In order to integrate the mathematical modeling thought into the teaching of this course, teachers must carefully design the teaching process so that the modeling thought can play a leading role. The purpose is to promote the mutual penetration of mathematical modeling thought and fuzzy 
mathematical knowledge, so that the teaching focus can be improved in the modeling process, thus forming a complete teaching system. Mathematics mainly explores and studies the objective world from the aspect of quantity, and any object or thing in the objective world is the unity of opposites between quality and quantity [8]. In order to ensure the smooth solution of practical problems, teachers should follow the evaluation method of mathematical modeling to establish clear and feasible problem evaluation methods. The teaching of mathematical thinking methods always depends on the teaching of mathematical knowledge, and the teaching of mathematical knowledge cannot be separated from mathematical thinking methods at all.

\section{Acknowledgement}

The authors acknowledge the Zhonghuan Information College Tianjin University of Technology, central foundation for young teachers' scientific research seedling. project name: the theory of fuzzy mathematics and coherent structures in the application of mathematical modeling. project (Grant:2019 kylg04).

\section{References}

[1] Zhao Huajie(2019). Air quality assessment method based on mathematical modeling [J]. Wireless Internet technology,No.8,pp.106-107.

[2] Wang Weiwei, Xu Anyang, Xin Yucui, et al(2016). Solution research and analysis of fuzzy comprehensive evaluation method in mathematical modeling [J]. Mathematics learning and research, No.15,pp.130-130.

[3] Wang Ruihua, Zhang Wenjuan, Chen Jianchu, et al(2015). Optimizing the process of braised pork based on the comprehensive evaluation of fuzzy mathematics [J]. Science and technology of food industry, Vol.36,No.6,pp.274-278.

[4] Sun Hongmei, Wang Jinzhi, Zhang Chunhui, et al(2014). Optimization of chicken bone element Maillard reaction process by fuzzy mathematics [J]. Chinese Journal of food science, Vol.14,No.6,pp.74-80.

[5] Qiao Yu, Liang Xiujuan, Wang Yubo, et al(2015). Application of improved fuzzy mathematics in groundwater quality assessment [J]. Hydropower and energy science,No.6,pp.27-30.

[6] Renhai, Ren Guangming, Zhang Tao, et al(2014). Slope stability evaluation of a hydropower station based on fuzzy mathematics [J]. Subgrade engineering, No.1,pp.143-147.

[7] Zhu Hongyan, Zhang Qiumei, Wang Guoli, et al(2015). Fuzzy mathematics comprehensive evaluation of the taste correction of compound Banlangen oral liquid [J]. Chinese Journal of experimental formulary, Vol.21,No.7,pp.8-10.

[8] Wang Xueqin, Liu Tongxing, Jiang Ziqi, et al(2014). Structural safety evaluation of bridges in service based on test detection and fuzzy mathematics theory [J]. Chinese and foreign highways, Vol.34,No.5,pp.147-151. 